\title{
Physical activity in COPD patients: patterns and bouts
}

\author{
David Donaire-Gonzalez $z^{1,2,3,4}$, Elena Gimeno-Santos $1,2,3$, Eva Balcells ${ }^{5,6,7}$, \\ Diego A. Rodríguez ${ }^{5,6,8}$, Eva Farrero9 , Jordi de Batlle $1,2,3$, Marta Benet ${ }^{1,2,3}$, \\ Antoni Ferrer ${ }^{5,6}$, Joan A. Barberà ${ }^{6,8}$, Joaquim Gea $5,6,7$, Robert Rodriguez-Roisin ${ }^{6,8}$, \\ Josep M. Antó ${ }^{1,2,3,7}$ and Judith Garcia-Aymerich ${ }^{1,2,3,7}$
}

Affiliations: ${ }^{1}$ Centre for Research in Environmental Epidemiology (CREAL), Barcelona, ${ }^{2}$ IMIM (Hospital del Mar Research Institute), Barcelona, ${ }^{3}$ CIBER Epidemiología y Salud Pública (CIBERESP). Barcelona, ${ }^{4}$ Physical Activity and Sports Sciences Dept, Fundació Blanquerna, Barcelona, ${ }^{5}$ Dept of Pneumology, Hospital del MarIMIM, Barcelona, ${ }^{6} \mathrm{CIBER}$ de Enfermedades Respiratorias (CIBERES), Barcelona, ${ }^{7}$ Dept of Experimental and Health Sciences, Universitat Pompeu Fabra, Barcelona, ${ }^{8}$ Dept of Pneumology, Hospital Clínic - Institut D'Investigacions Biomèdiques August Pi i Sunyer (IDIBAPS), Universitat de Barcelona, Barcelona, and ${ }^{9}$ Servei de Pneumologia, Hospital Universitari de Bellvitge IDIBELL (Hospital de Bellvitge Research Institute), Barcelona, Spain.

Correspondence: J. Garcia-Aymerich, Centre for Research in Environmental Epidemiology (CREAL), Dr. Aiguader 88, 08003 Barcelona, Spain. E-mail: jgarciaAcreal.cat

ABSTRACT The present study aims to describe the pattern of physical activity and the frequency, duration and intensity of physical activity bouts in patients with chronic obstructive pulmonary disease (COPD), to assess how these patterns differ according to COPD severity, and to explore whether these patients meet the general guidelines for physical activity for older adults.

177 patients $(94 \%$ male, mean \pm SD age $71 \pm 8$ years and forced expiratory volume in $1 \mathrm{~s} 52 \pm 16 \%$ predicted) wore the SenseWear $\mathrm{Pro}_{2}$ Armband accelerometer for eight consecutive days. Physical activity bouts were defined as periods of $\geqslant 10 \mathrm{~min}$ above 1.5 metabolic equivalent tasks and classified according to their median intensity.

Patients engaged in activity a median of $153 \mathrm{~min} \cdot \mathrm{day}^{-1}$ and $57 \%$ of that time was spent in bouts. Median frequencies of bouts per day were four and three for all and moderate-to-vigorous intensities, respectively. With increasing COPD severity, time in physical activity, proportion of time in bouts and frequency of bouts decreased. $61 \%$ of patients fulfilled the recommended physical activity guidelines.

In conclusion, COPD patients of all spirometric severity stages engage in physical activity bouts of moderate-to-vigorous intensities. Patients with severe and very severe COPD perform their daily activities in fewer and shorter bouts than those in mild and moderate stages.

@ERSpublications Patients with severe COPD perform their daily activities in fewer, shorter bouts than those in mild and moderate stages http://ow.ly/nug7k

This article has supplementary material available from www.erj.ersjournals.com

Received: July 022012 | Accepted after revision: Nov 292012 | First published online: Dec 202012

Conflict of interest: Disclosures can be found alongside the online version of this article at www.erj.ersjournals.com Copyright @ERS 2013 


\section{Introduction}

Among patients with chronic obstructive pulmonary disease (COPD), reduced levels of physical activity have been found to be related to an increased risk of hospital admissions and mortality [1-3]. In addition, research on the physical activity levels of COPD patients has consistently shown that COPD patients have lower physical activity levels than their healthy peers [4, 5]. Most previous studies have reported accurate measurements of the physical activity level without addressing the pattern of activity. Indeed, physical activity is recognised as a multi-faceted behaviour that involves frequency, intensity, time and type (FITT principle) as modifiable components that are specifically used for guiding and testing interventions $[6,7]$. Consistent with this concept, the recommendations from the American College of Sports Medicine and the American Heart Association advise that older adults "should perform moderate-intensity aerobic (endurance) physical activity for a minimum of $30 \mathrm{~min}$ on 5 days each week or vigorous-intensity aerobic activity for a minimum of $20 \mathrm{~min}$ on 3 days each week" [8]. Importantly, current physical activity guidelines explicitly acknowledge that the recommendations should be used in the context of the subject's needs, goals and initial abilities; thus, the 30 consecutive minutes of activity could be replaced by two or three bouts of at least $10 \mathrm{~min}$ each [9]. This adaptation is especially useful for COPD patients, given their limitations engaging in more activity [10]. Unfortunately, the lack of information on the pattern of physical activity in COPD patients with respect to the bouts of activity may have limited our ability to design interventions with realistic goals for this population [11]. For patients with other diseases, such as arterial hypertension, information on bout frequency, duration and intensity has enabled the development of recommendations for the primary prevention, treatment and control of this condition [12]. The present study aims to describe the pattern of physical activity and the frequency, duration and intensity of physical activity bouts in patients with COPD, to assess how these patterns differ according to COPD severity, and to explore whether these patients meet the general guidelines for physical activity for older adults.

\section{Methods}

\section{Participants}

This study is part of the Phenotype and Course of Chronic Obstructive Pulmonary Disease (PAC-COPD) cohort. Patients with a diagnosis of COPD (ratio of the post-bronchodilator forced expiratory volume in $1 \mathrm{~s}$ $(\mathrm{FEV} 1)$ to the forced vital capacity $(\mathrm{FVC})<70 \%)[13]$ were recruited at nine tertiary hospitals in Spain [14, 15] and their spirometric severity was classified according to American Thoracic Society/European Respiratory Society criteria [13]. Of the 342 COPD patients included in the PAC-COPD cohort, 177 patients had physical activity data available and were, therefore, included in the present analysis. There were no differences between these patients and the remaining PAC-COPD patients, as previously reported [16]. The study was approved by the ethics committees of all of the participating hospitals and written informed consent was obtained from all of the subjects.

\section{Variables and instruments of measurement}

All study tests were carried out on patients in clinically stable conditions at least 3 months after the last recorded exacerbation. Physical activity levels and bouts, and adherence to recommendations were measured using the SenseWear $\mathrm{Pro}_{2}$ Armband accelerometer (Body Media, Pittsburgh, PA, USA), which has proven a valid tool to measure physical activity in COPD patients [17]. The accelerometer was worn for eight consecutive days and the minimal time was defined, a priori, as at least 3 days recording more than $70 \%$ of daily time (08:00-22:00 h) [18]. The consistency of accelerometer data was tested by the intra-class correlation coefficient (ICC) of steps per day between all possible combinations of 3 days (ICC 0.95, 95\% CI 0.93-0.96). The accelerometer was worn on the right arm and recorded the subjects' movements from lower and upper body. More details on the accelerometer wearing time and recording have been previously published [16].

Support statement: Supported by Fondo de Investigación Sanitaria (FIS PI052292) and Spanish Society of Pneumology and Thoracic Surgery (SEPAR 2004/136). J. Garcia-Aymerich has a researcher contract from the Instituto de Salud Carlos III (CP05/00118), Ministry of Health, Spain. J. de Batlle had a predoctoral fellowship from the Instituto de Salud Carlos III (FI05/01022), Ministry of Health, Spain. D.A. Rodríguez has a long-term research fellowship from the European Respiratory Society (2006/191). The PAC-COPD Study is funded by grants from Fondo de Investigación Sanitaria (FIS PI020541), Ministry of Health, Spain; Agència d'Avaluació de Tecnologia i Recerca Mèdiques (AATRM 035/20/02), Catalonia Government; Spanish Society of Pneumology and Thoracic Surgery (SEPAR 2002/137); Catalan Foundation of Pneumology (FUCAP 2003 Beca Marià Ravà); Red RESPIRA (RTIC C03/11); Red RCESP (RTIC C03/09), Fondo de Investigación Sanitaria (PI052486); Fondo de Investigación Sanitaria (PI052302); Fundació La Marató de TV3 (number 041110); DURSI (2005SGR00392); and an unrestricted educational grant from Novartis Farmacèutica, Spain. CIBERESP and CIBERES are funded by the Instituto de Salud Carlos III, Ministry of Health, Spain. No involvement of funding sources in study design; in the collection, analysis, and interpretation of data; in the writing of the report; or in the decision to submit the article for publication. The researchers are independent from the funders. 
The accelerometer provided a minute-by-minute report for each subject, with values for the number of steps and the metabolic equivalent tasks (METs) that were used to compute the variables of interest.

First, the physical activity was characterised using energy expenditure $\left(\mathrm{MET} \cdot \mathrm{min} \cdot \mathrm{day}^{-1}\right)$, time $\left(\mathrm{min} \cdot \mathrm{day}^{-1}\right)$, intensity (METs), time spent in minutes with $\geqslant 1.5 \mathrm{MET}$ and steps (steps per day). Secondly, physical activity bouts were defined as any period of at least $10 \mathrm{~min}$ with an intensity $\geqslant 1.5$ MET and classified as light, moderate or vigorous according to their median intensity (in METs). The thresholds for moderate and vigorous physical activity were set at $50 \%$ and $65 \%$ of the maximum oxygen consumption [19] from an incremental test (mean $16 \mathrm{~mL} \cdot \mathrm{min}^{-1} \cdot \mathrm{kg}^{-1}$, reported elsewhere) [15], following the most recent recommendations on the best practices for the use of objective methods to assess physical activity in studies for adults with functional limitations [20], and resulted in 2.6 and 3.4 MET, respectively [19]. We computed the frequency (bouts per day), duration (minutes per bout), intensity (METs) and total time (min $\cdot$ day $^{-1}$ ) in bouts. To exemplify the latter, figure 1 shows a minute-by-minute METs report from the accelerometer in a sample day of a COPD patient, together with the graphical illustration of six physical activity bouts and their duration and median intensity. The ratio of time in physical activity bouts to time in physical activity was also obtained. Thirdly, the adherence to the recommendation for older adults was defined as spending $\geqslant 30 \mathrm{~min}$ in moderate activity $\geqslant 5$ days per week or spending $\geqslant 20 \mathrm{~min}$ in vigorous activity $\geqslant 3$ days per week [8]. For both definitions, we distinguished if the minimum time spent in activity was achieved through consecutive minutes or through the accumulation of bouts.

Self-reported physical activities were obtained with the Spanish version of the Yale Physical Activity Survey [21], as previously validated for our COPD population [16]. This questionnaire collects information

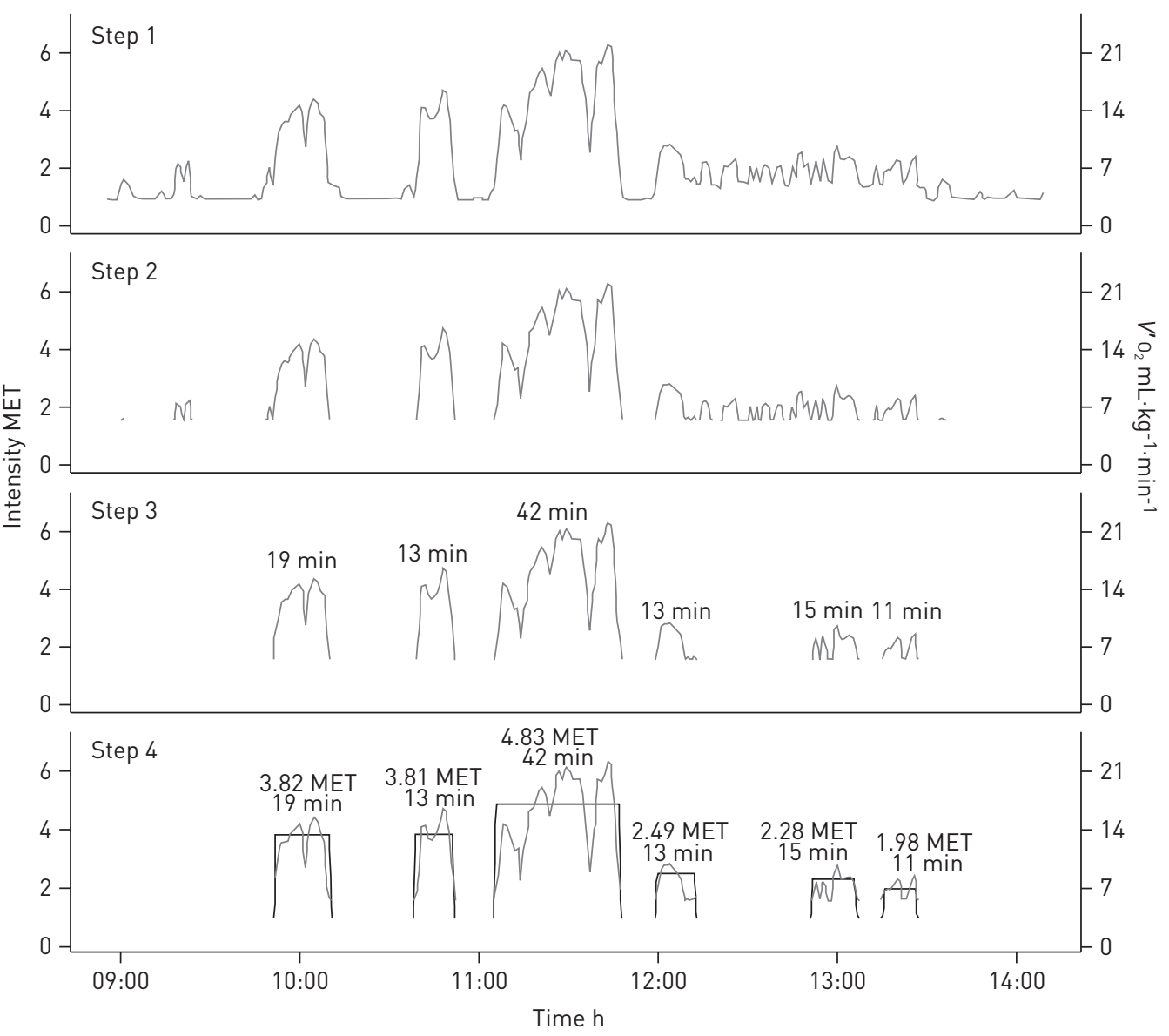

FIGURE 1 Graphical illustration of the process to identify physical activity bouts from the minute-by-minute metabolic equivalent tasks (METs) report of the accelerometer during a sample day in a chronic obstructive pulmonary disease patient. The continuous grey line represents the minute-by-minute MET values recorded by the accelerometer. Step 1 (if required): to format data into the minute-by-minute report of MET values; step 2: to keep minutes in which activity is $\geqslant 1.5$ MET; step 3: to calculate the duration of consecutive minutes and keep periods $\geqslant 10 \mathrm{~min}$; step 4: to calculate the average intensity of each bout, to be classified into light, moderate or vigorous according to each population threshold. $V^{\prime} \mathrm{O}_{2}$ : oxygen uptake. 
(frequency and duration) on a wide range of activities performed in a typical week of the previous month. Other relevant variables included sociodemographic factors, smoking, Charlson comorbidity index, dyspnoea, the St George's Respiratory Questionnaire, lung function measurements (post-bronchodilator FEV1, FVC and FEV1/FVC, residual volume (RV), total lung capacity (TLC), RV/TLC, and arterial oxygen tension, body mass index, fat-free mass index and the 6-min walking distance. Details of these procedures have been published previously $[14,15]$.

\section{Statistical analysis}

Sample size calculations are available in the online supplementary material. The subjects' characteristics and physical activity characteristics are presented as $\mathrm{n}(\%)$ for categorical variables and mean \pm SD or median (interquartile range) for continuous variables with normal and non-normal distributions, respectively. Physical activity variables were modelled using Poisson regression. Tests for trends across COPD severity stages were obtained by treating the COPD severity stages as a continuous variable. For sensitivity analyses, we repeated all analyses: 1) using standard cut-off points for intensity of physical activity (3 MET for moderate and 6 MET for vigorous physical activity [9]); and 2) excluding subjects with extreme values (above the 95th percentile) in the number of daily steps recorded by the accelerometer. All analyses were conducted using R 2.14.1 (the R Project for Statistical Computing, www.r-project.org). The scripts for the calculation of bouts are available in the online supplementary material.

\section{Results}

Table 1 shows the main characteristics of the patients. Patients wore the accelerometer for a mean of 6 days and recorded a mean of $95 \%$ of daily time (13.5 h of 14 -h maximum). Almost all COPD patients $(98 \%)$ participated in physical activity bouts on a daily basis and $57 \%$ of their physical activity was performed in bouts (table 2). Median number of daily bouts was 4.4 and 2.6 for all intensities and moderate-to-vigorous intensities, respectively. The median duration of the bouts was $\sim 20 \mathrm{~min}$, irrespective of their intensity. Overall, our patients exhibited moderate exercise limitation and were reasonably active. Exercise capacity and physical activity were moderately correlated (Spearman $\mathrm{r}=0.54 ; \mathrm{p}<0.001$ ) (online supplementary fig. S1).

TABLE 1 Sociodemographic and clinical characteristics according to levels of chronic obstructive pulmonary disease (COPD) severity

$\begin{array}{llll}\begin{array}{l}\text { All COPD } \\ \text { patients }\end{array} & \text { Mild COPD } \quad \text { Moderate COPD } \quad \text { Severe COPD } & \text { Very Severe COPD } \\ \end{array}$

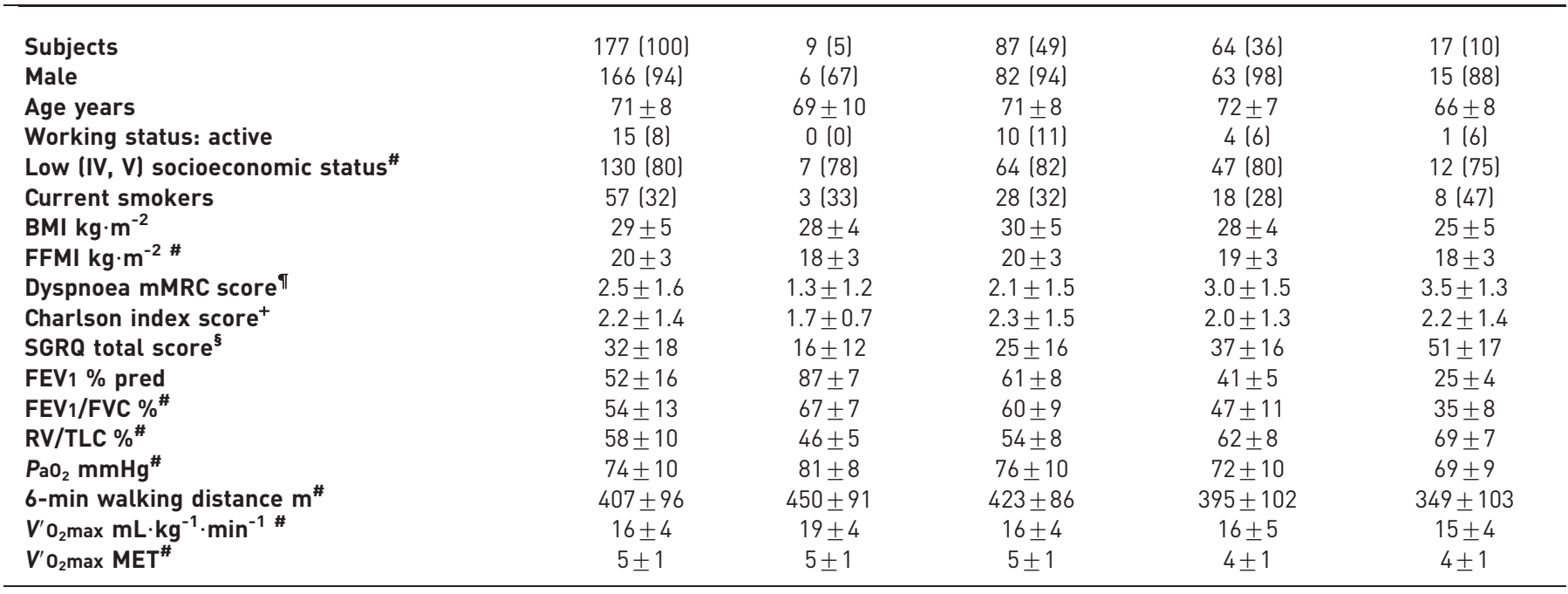

Data are presented as $\mathrm{n}(\%)$ or mean \pm SD. BMI: body mass index; FFMI: fat-free mass index; mMRC: modified Medical Research Council; SGRQ: St George's Respiratory Questionnaire; FEV1: forced expiratory volume in 1 s; \% pred: \% predicted; FVC: forced vital capacity; RV: residual volume; TLC: total lung capacity; $\mathrm{PaO}_{2}$ : arterial oxygen tension; $V^{\prime} \mathrm{O}_{2}$ max: maximal oxygen uptake; MET: metabolic equivalent task. "\# : some values are missing for certain variables (15 in socioeconomic status, 13 in FFMI, one in FEV1/FVC, 11 in RV/TLC, six in $P_{a O_{2}}, 10$ in 6-min walking distance and 66 in $V^{\prime} \mathrm{O}_{2}$ max); missing values were distributed at random and were mainly due to the hospital logistics and patients' availability, as previously published [15]. ': out of five; ${ }^{+}$: out of $30 ;{ }^{\S}$ : out of 100 . 
TABLE 2 Characteristics of physical activity and physical activity bouts in chronic obstructive pulmonary disease patients

All intensities ( $\geqslant 1.5 \mathrm{MET}$ )

\section{Characteristics of physical activity}

Steps per day

Energy expenditure in physical activity MET $\cdot \min \cdot$ day $^{-1}$

Time in physical activity $\min \cdot$ day $^{-1}$

Intensity of physical activity MET

\section{Characteristics of physical activity bouts}

Participation in physical activity bouts $\mathrm{n}(\%)$

Frequency bouts $\cdot$ day $^{-1 \%}$

Duration $\mathrm{min} \cdot$ bout $^{-1}$ "1

Intensity MET

Time in bouts $\mathrm{min} \cdot \mathrm{day}^{-1}$

Time in bouts out of total time in activity \%"
Moderate-to-vigorous intensities

$(\geqslant 2.6 \mathrm{MET})^{\#}$

Data are presented as median (interquartile range), unless otherwise stated. $n=177$ patients. MET: metabolic equivalent task; ${ }^{\text {: }}$ cut-off points for definition of intensity of physical activity are based on the mean values of maximal oxygen uptake at cardiopulmonary incremental exercise test peak in this population (see methods). ": the frequency, duration, intensity, time in bouts and time in bouts out of total time in activity were only computed for subjects that had at least one bout in the whole recording period.
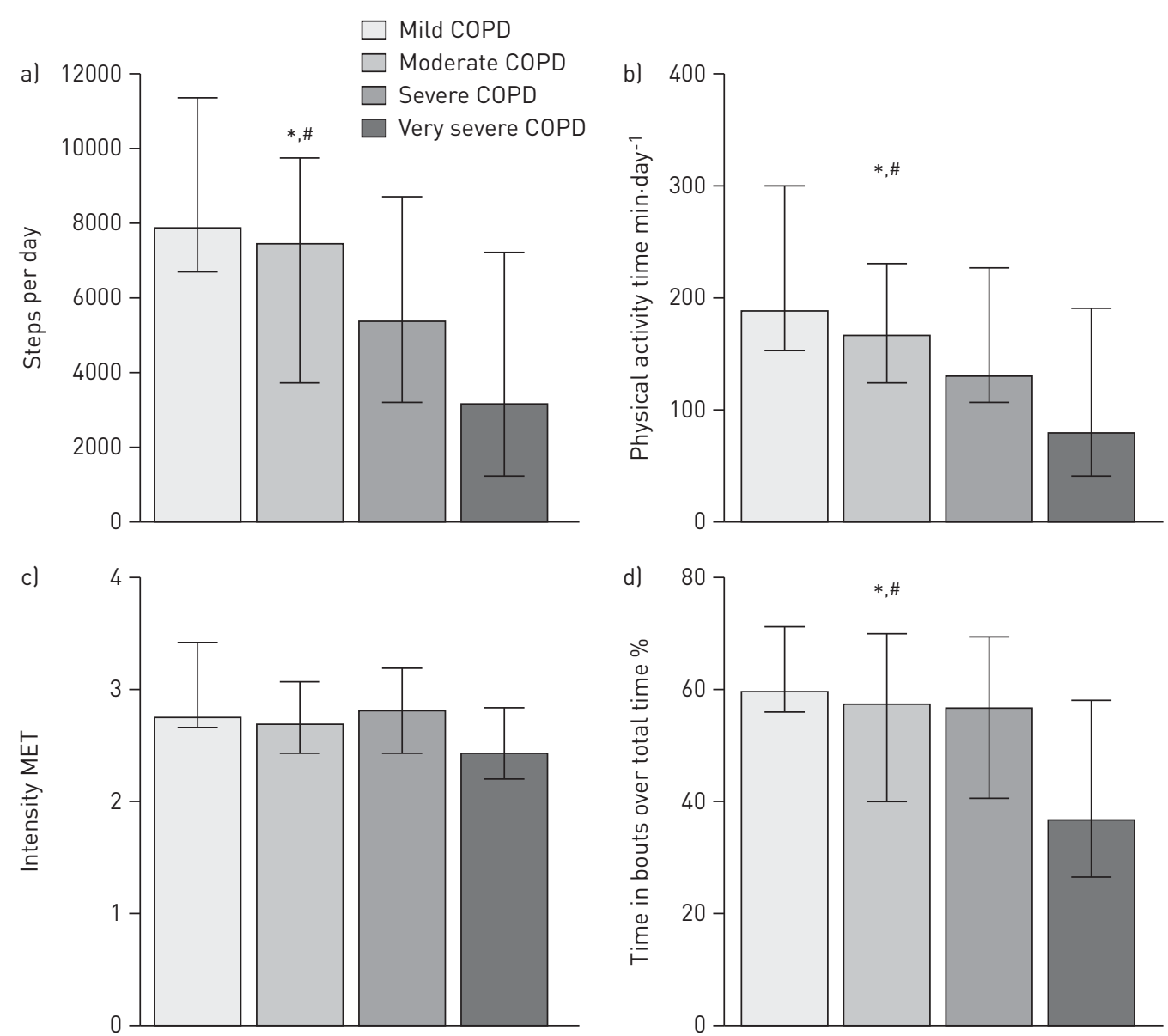

FIGURE 2 Characteristics of physical activity across levels of chronic obstructive pulmonary disease (COPD) severity. Bar plots represent the medians and error bars the interquartile range. a) Total number of steps per day, b) total time in physical activity $\geqslant 1.5$ metabolic equivalent tasks (METs), c) mean intensity in physical activity and d) percentage of time in physical activity bouts out of the total time in physical activity. ${ }^{*}$ : $p$-trend across COPD severity stages $<0.05$; $^{*}:$-value comparing mild-to-moderate versus severe-to-very-severe COPD $<0.05$. 
Figure 2 shows that the number of steps, time in physical activity and proportion of time in bouts over total time in physical activity exhibited a significant, steady decrease with increasing COPD severity, but no differences in intensity were found. The frequency of the bouts, as well as the total time spent in bouts, decreased with increasing COPD severity (fig. 3). Patients with severe-to-very-severe COPD reported lower participation and less time spent in some of the leisure time activities (gardening and exercising) compared to those with mild-to-moderate COPD, while there were no differences in participation and time spent in household activities or recreational activities (online supplementary table S1).
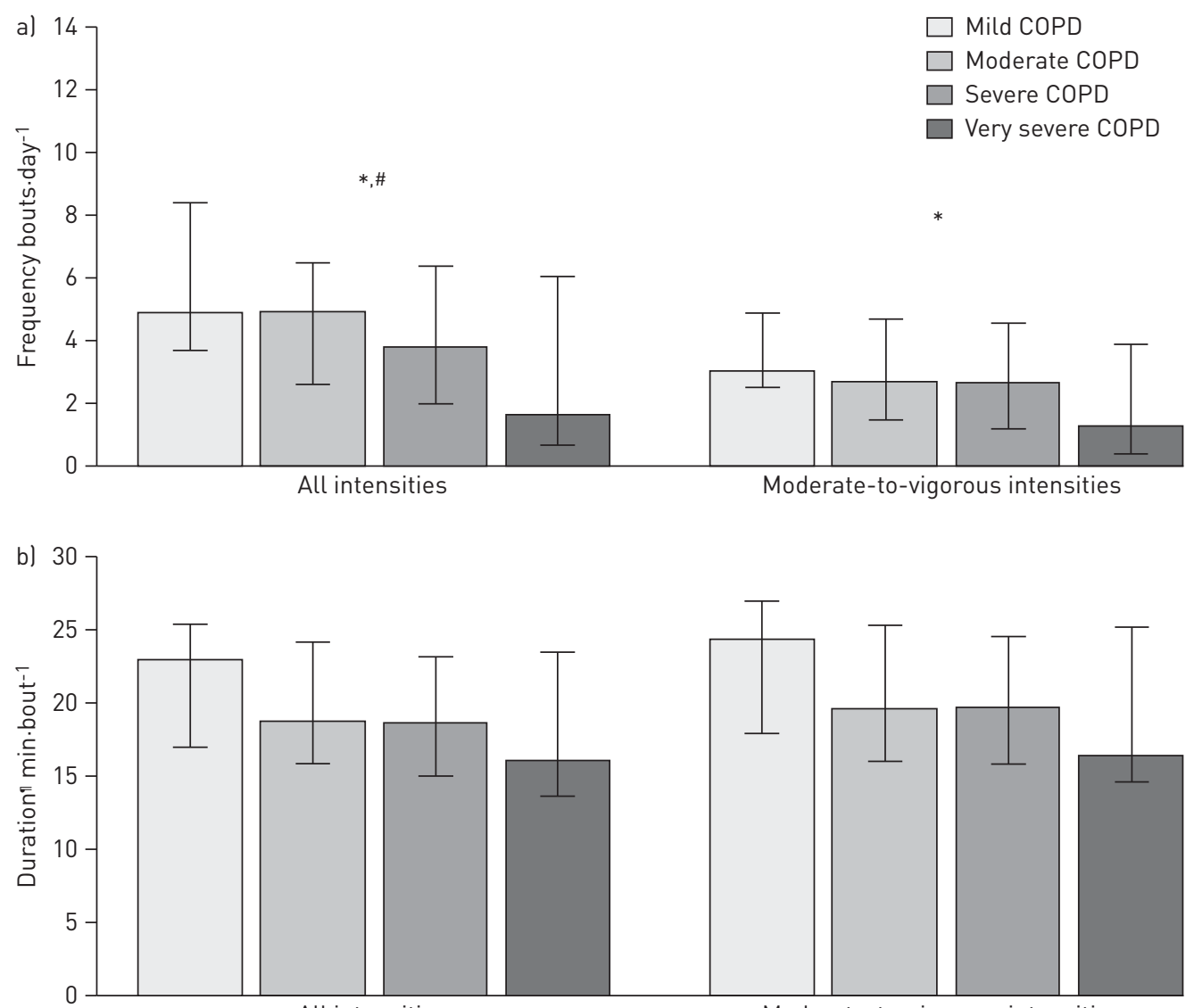

All intensities

Moderate-to-vigorous intensities

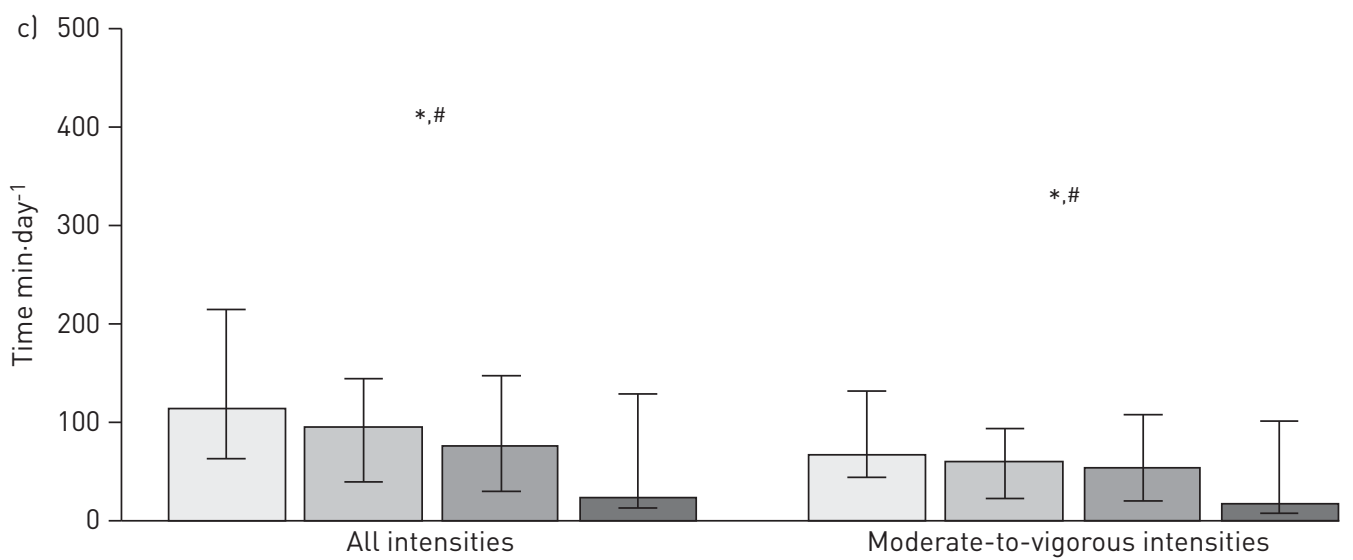

FIGURE 3 Frequency, duration and time in physical activity bouts of all and moderate-to-vigorous intensities, across levels of chronic obstructive pulmonary disease (COPD) severity. Bar plots represent the medians and error bars the interquartile range. a) Number of physical activity bouts per day, b) mean duration of the physical activity bouts and c) total amount of time in physical activity bouts. ${ }^{*}$ : p-trend across COPD severity stages $<0.05$; ${ }^{\sharp}$ : p-value comparing mildto-moderate versus severe-to-very-severe COPD $<0.05$; ${ }^{\circ}$ : only patients who participated in bouts. 

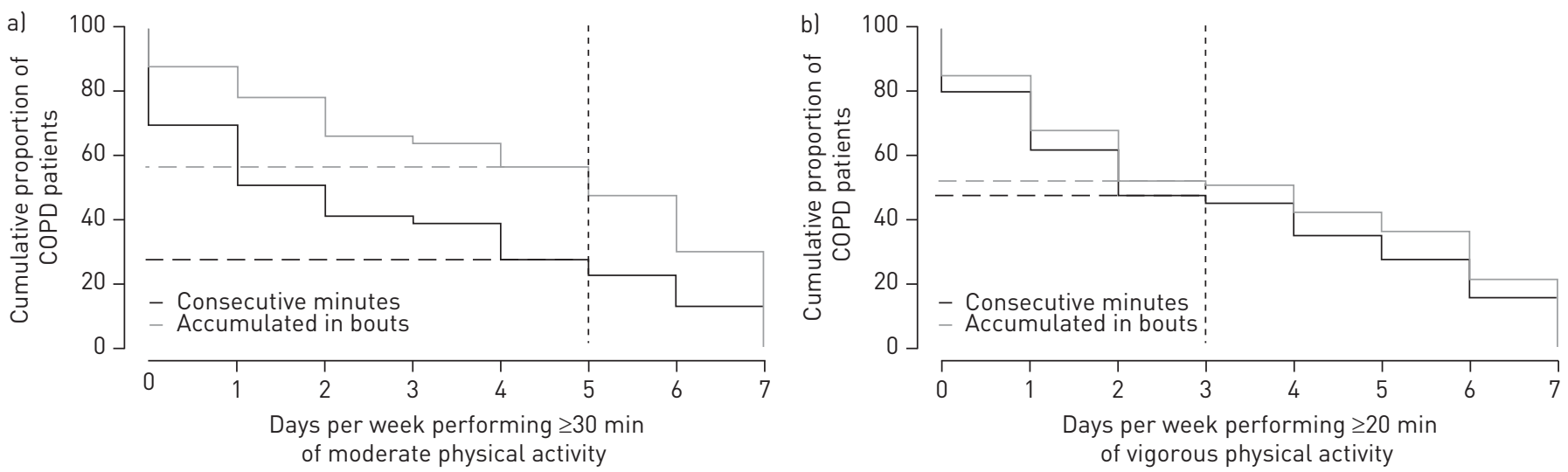

c)

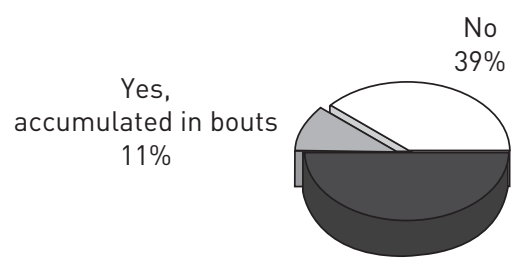

Yes, consecutive minutes $50 \%$
FIGURE 4 Chronic obstructive pulmonary disease (COPD) patients' adherence to physical activity recommendations for older adults, i.e. moderate-intensity physical activity for $\geqslant 30 \mathrm{~min}$ on 5 days each week or vigorous-intensity aerobic activity for $\geqslant 20 \mathrm{~min}$ on 3 days each week [8]. a) Days per week performing $\geqslant 30 \mathrm{~min}$ of moderate physical activity, b) days per week perfoming $\geqslant 20 \mathrm{~min}$ of vigorous physical activity and c) adherence to physical activity recommendations for the elderly.

Figure 4 shows that about $25 \%$ of the COPD patients fulfilled the recommendation of engaging in $\geqslant 30$ consecutive minutes of moderate physical activity five or more days per week. The proportion increased to almost $60 \%$ when the duration of $\geqslant 30$ min per day was achieved through the accumulation of bouts of $\geqslant 10$ min duration. Overall, $61 \%$ of COPD patients fulfilled the physical activity recommendation for older adults. Table 3 shows that the patients who adhered to this recommendation had reported higher time in leisure activity, primarily yard-work or gardening and recreational activities.

All analyses were repeated using standard cut-off points for the definition of moderate and vigorous intensities of physical activity (online supplementary tables S2 and S3 and figs S2 and S3). Results for participation, frequency, duration and intensity of bouts were very similar. The proportion of COPD

TABLE 3 Self-reported (Yale Physical Activity Survey) time spent weekly in physical activities, according to the adherence to physical activity recommendation for older adults ${ }^{\#}$

Adherence to physical activity recommendation

p-value ${ }^{\pi}$

\begin{tabular}{cc}
\hline No & $\begin{array}{c}\text { Yes, accumulated in } \\
\geqslant 10 \mathrm{~min}\end{array}$ \\
$66(39)$ & $17(10)$ \\
$8.1 \pm 8.1$ & $6.3 \pm 7.1$ \\
$3.7 \pm 11.8$ & $2.4 \pm 9.7$ \\
$11.6 \pm 11$ & $16.2 \pm 13.4$ \\
$1.1 \pm 4.3$ & $4.1 \pm 6.9$ \\
$0.9 \pm 4.5$ & $0 \pm 0$ \\
$0.7 \pm 1.5$ & $2.5 \pm 7.6$ \\
$9 \pm 10$ & $9.6 \pm 9.6$
\end{tabular}

Yes, in consecutive minutes

Subjects $\mathbf{n}(\%)$
Household activities $\mathbf{h} \cdot$ week $^{-1}$
Work activities $\mathbf{h} \cdot$ week $^{-1}$
Leisure time activities $\mathbf{h} \cdot$ week $^{-1}$
Yard work/gardening $\mathrm{h} \cdot$ week $^{-1}$
Caretaking $\mathrm{h} \cdot$ week $^{-1}$
Exercise $\mathrm{h} \cdot$ week $^{-1}$
Recreational activities $\mathrm{h} \cdot$ week $^{-1}$

Data are presented as mean $\pm S D$, unless otherwise stated. ${ }^{\#}$ : moderate-intensity physical activity for $\geqslant 30$ min on 5 days each week or vigorousintensity aerobic activity for $\geqslant 20 \mathrm{~min}$ on 3 days each week [8]; ${ }^{\uparrow}$ : Kruskal-Wallis test to compare the three groups of adherence to recommendations. 
patients fulfilling the physical activity recommendation was reduced from $61 \%$ to $50 \%$ (online supplementary fig. S3). Sensitivity analysis excluding subjects with extreme values yielded notably similar results.

\section{Discussion}

This report is the first assessing bouts of physical activity in COPD patients. It shows that these subjects are able to perform bouts of moderate-to-vigorous physical activity. The severity of COPD is inversely associated with the frequency of bouts. More than $60 \%$ of our COPD patients fulfilled the physical activity recommendation for older adults.

\section{Comparison with previous studies}

Our results on the patterns of physical activity can be compared with previous research. The COPD patients included in our study walked more (according to steps per day or walking time measures) than COPD patients who participated in previous studies from four different geographic locations [18, 22, 23]. Our patients were older than, and had similar airflow limitation and exercise capacity to patients in these former studies. Interestingly, the time spent in activity was similar across studies, suggesting a higher participation, duration or speed during walking activities in our population. In fact, a previous study found that COPD patients walk $25 \%$ slower than healthy age-matched controls [23]. Akin to this finding, only $7 \%$ of the walking time in our patients was reported as brisk walking, as compared to $75 \%$ in a study with healthy subjects of similar age and geographical location using an identical physical activity questionnaire [24]. Another difference between previous studies and ours is that the earlier studies recruited patients with an established diagnosis of COPD from outpatient clinics, whereas our sample was made up of patients who were recruited after their first COPD admission, one-third of whom were undiagnosed as COPD [14]. Thus, the limiting effects of COPD on activity may be more related to the specific time-point in the course of the disease, rather than to its spirometric severity.

Our results, consistent with previous data that showed that the severity of airflow limitation is related to the level of physical activity $[4,5]$, provide, for the first time, information on the activity pattern that is behind the differences in levels. Our very severe COPD patients spent a lower proportion of their activity time in bouts, at the expense of reducing bout frequency (fig. 2). The lack of differences in bout duration across severity stages needs to be interpreted with caution; the very severe COPD patients perform bouts that have similar duration to those of patients in other severity stages. However, the lower proportion of time spent in bouts implies that their physical activity is performed in episodes of $<10 \mathrm{~min}$ duration. The lack of differences in intensity of physical activity across severity stages is well known. It has been hypothesised that COPD patients increase the intensity of their daily physical activities as a result of "trying to perform activities as fast as possible so as to alleviate the unease caused by physical activity" [5]. In our study, patients kept doing both household and recreational activities; the former are likely practised despite disease severity because they are compulsory for daily living, while the latter, more specifically in the case of leisure walking, because they are perceived as healthy, social or simply pleasant. Altogether our findings suggest that the exercise limitation in COPD primarily affects the way patients distribute their periods of activity over time, towards fewer and shorter episodes of uninterrupted activity.

The proportion of COPD patients meeting the physical activity recommendation has been reported to be an important subject for COPD research [10] that had not previously been assessed. We approached this subject by using an objective tool for defining bouts in agreement with the definitions of the international physical activity guidelines, which are widely known, disseminated and implemented in Spain. The comparison with findings from other studies $[25,26]$ should consider the current evidence that geographical, cultural and lifestyle factors affect the practice of physical activity.

\section{Applicability of the results}

There is an emerging need for interventions that aim to increase the physical activity level of COPD patients $[10,27]$. These interventions include, but are not restricted to, pulmonary rehabilitation and/or community health promotion programmes and should be considered in addition to the promotion of the light intensity activities frequently performed during daily life [8]. Our detailed assessment of physical activity patterns provides important insight into the design of such interventions. First, the proportion of patients achieving the recommended physical activity levels is elevated when shorter bouts are grouped together, rather than when definitions are restricted to consecutive minutes. Thus, fulfilling the recommendation with short bouts may be more feasible and not necessarily less effective because several clinical trials have demonstrated similar effects in aerobic fitness, weight loss, and other cardiovascular risk factors with either long-bout $(\geqslant 20 \mathrm{~min}$ ) or short-bout $(\geqslant 10 \mathrm{~min}$ ) interventions [28-30]. Interestingly, in our study, patients fulfilling the recommendation also reported more time engaged in recreational activities. Secondly, it is interesting to note that the way COPD patients seem to adapt to exercise limitation (towards less and shorter periods of 
activity, as discussed above) matches with the strategy of interval training that is used in pulmonary rehabilitation, specifically in the most severe patients. We suggest that a meaningful focus of rehabilitation programmes is increasing the frequency of bouts rather than prolonging the duration of current bouts. This is supported by a previous 6-month pulmonary rehabilitation programme, which found that the increase in time spent walking after the intervention was due to an increase in the frequency of short activity blocks $(<1 \mathrm{~min})$ rather than to a lengthening of existing periods [31]. Finally, the differing physical activity levels observed between the patients in the current study and the patients from other studies with similar COPD severities or other populations suggest that interventions designed for COPD patients should not only be based on pathophysiology-related limitations but also on the "subjects' needs, goals, and initial abilities", as advocated by the recommendations $[8,9]$.

\section{Limitations and strengths}

The current study has several limitations. The cross-sectional design does not allow for the differences to be interpreted across COPD severity stages, such as the evolution of physical activity over time. The lack of a control (non-COPD) group may be seen as a limitation. However, because the aim of the project was to describe the characteristics of physical activity in COPD patients, we believe that the population addresses the needs of the research question. Unfortunately, the small number of patients in the mild and very severe COPD groups could have led to reduced statistical power to identify as statistically significant the observed differences in physical activity. Despite this fact, our study still includes a considerable number of patients with moderate and severe COPD, which is more than in previous COPD studies [5]. We defined a bout as a minimum of 10 consecutive minutes in activity, according to public health recommendations [32, 33], a definition that has not been consistently applied as yet, such that the comparison of our results with existing research is currently limited. Finally, our findings on physical activity levels and patterns may not be applicable to other COPD groups with more impaired exercise capacity or in other geographical areas, which may differ with respect to climate or lifestyle. However, the relationship between activity levels and patterns with disease severity is still valid and original.

One strength of our study is the large number of patients who were assessed using accelerometry during a period of eight consecutive days. Other physical activity instruments or shorter recording periods would have not allowed for the calculation of weekly patterns of physical activity bouts. The fact that all patients were recruited at the same time-point during the clinical course of COPD allows for the avoidance of the potential confounding factors produced by changes during the course of disease. Finally, we defined the MET cut-off points for moderate and vigorous physical activity according to the mean maximal oxygen uptake during a cardiopulmonary incremental exercise test in the same patients $[15,19]$. Setting the cut-off points in this manner is a clear advantage in comparison with previous COPD research that used only the standard intensity cut-offs for the general population, which results in higher relative intensities when applied to older and less fit individuals $[19,20]$.

\section{Conclusions}

In conclusion, COPD patients of all spirometric severity stages engage in physical activity bouts of moderate-to-vigorous intensities. Patients with severe and very severe COPD perform their daily activities in fewer and shorter bouts than those in mild and moderate stages. Interventions that aim to increase the physical activity levels of COPD patients, including physical activity advice and pulmonary rehabilitation programmes, should focus on maximising endurance capacity of the patients.

\section{Acknowledgements}

The authors acknowledge E. Gracia and D. Martinez (Centre for Research in Environmental Epidemiology (CREAL), Barcelona, Spain) for their help in extracting data from the accelerometers. This manuscript was reviewed by two professional medical editors, Mark C and Sarah B, from American Journal Experts.

The Phenotype and Course of COPD (PAC-COPD) Study Group: Centre for Research in Environmental Epidemiology (CREAL), Barcelona: J.M. Antó (principal investigator), J. Garcia-Aymerich (project coordinator), M. Benet, J. de Batlle, I. Serra, D. Donaire-Gonzalez, S. Guerra; Hospital del Mar-IMIM, Barcelona: J. Gea (centre coordinator), E. Balcells, À. Gayete, M. Orozco-Levi, I. Vollmer; Hospital Clínic-Institut D’Investigacions Biomèdiques August Pi i Sunyer (IDIBAPS), Barcelona: J.A. Barberà (centre coordinator), F.P. Gómez, C. Paré, J. Roca, R. Rodriguez-Roisin, À. Agustí, X. Freixa, D.A. Rodriguez, E. Gimeno-Santos, K. Portillo; Hospital General Universitari Vall D’Hebron, Barcelona: J. Ferrer (centre coordinator), J. Andreu, E. Pallissa, E. Rodríguez; Hospital de la Santa Creu i Sant Pau, Barcelona: P. Casan (centre coordinator), R. Güell, A. Giménez; Hospital Universitari Germans Trias i Pujol, Badalona: E. Monsó (centre coordinator), A. Marín, J. Morera; Hospital Universitari de Bellvitge, Institut d'Investigació Biomèdica de Bellvitge (IDIBELL), L'Hospitalet de Llobregat: E. Farrero (centre coordinator), J. Escarrabill; Hospital de Sabadell, Corporació Parc Taulí, Institut Universitari Parc Taulí (Universitat Autònoma de Barcelona), Sabadell: A. Ferrer (centre coordinator); Hospital Universitari Son Dureta, Palma de Mallorca: J. Sauleda (centre coordinator), B. Togores; Hospital Universitario de Cruces, UPV, Barakaldo: J.B. Gáldiz (centre coordinator), L. López; Instituto Nacional de Silicosis, Oviedo: J. Belda. 


\section{References}

1 Garcia-Aymerich J, Lange P, Benet M, et al. Regular physical activity reduces hospital admission and mortality in chronic obstructive pulmonary disease: a population based cohort study. Thorax 2006; 61: 772-778.

2 Pitta F, Troosters T, Probst VS, et al. Physical activity and hospitalization for exacerbation of COPD. Chest 2006; 129: $536-544$

3 Waschki B, Kirsten A, Holz O, et al. Physical activity is the strongest predictor of all-cause mortality in patients with COPD: a prospective cohort study. Chest 2011; 140: 331-342.

4 Bossenbroek L, de Greef MH, Wempe JB, et al. Daily physical activity in patients with chronic obstructive pulmonary disease: a systematic review. COPD 2011; 8: 306-319.

5 Vorrink SN, Kort HS, Troosters T, et al. Level of daily physical activity in individuals with COPD compared with healthy controls. Respir Res 2011; 12: 33.

6 Barisic A, Leatherdale ST, Kreiger N. Importance of frequency, intensity, time and type (FITT) in physical activity assessment for epidemiological research. Can J Public Health 2011; 102: 174-175.

7 US Dept of Health and Human Services. Physical Activity and Health: A Report of the Surgeon General. Atlanta, US Dept of Health and Human Services, Centers for Disease Control and Prevention, 1996. Available from www. cdc.gov/nccdphp/sgr/pdf/sgrfull.pdf

8 Nelson ME, Rejeski WJ, Blair SN, et al. Physical activity and public health in older adults: recommendation from the American College of Sports Medicine and the American Heart Association. Med Sci Sports Exerc 2007; 39: 1435-1445.

9 Garber CE, Blissmer B, Deschenes MR, et al. American College of Sports Medicine position stand. Quantity and quality of exercise for developing and maintaining cardiorespiratory, musculoskeletal, and neuromotor fitness in apparently healthy adults: guidance for prescribing exercise. Med Sci Sports Exerc 2011; 43: 1334-1359.

10 Troosters T, Gosselink R, Janssens W, et al. Exercise training and pulmonary rehabilitation: new insights and remaining challenges. Eur Respir Rev 2010; 19: 24-29.

11 Miller CK, Headings A, Peyrot M, et al. Goal difficulty and goal commitment affect adoption of a lower glycemic index diet in adults with type 2 diabetes. Patient Educ Couns 2012; 86: 84-90.

12 Pescatello LS, Franklin BA, Fagard R, et al. American College of Sports Medicine position stand. Exercise and hypertension. Med Sci Sports Exerc 2004; 36: 533-553.

13 Celli BR, MacNee W. Standards for the diagnosis and treatment of patients with COPD: a summary of the ATS/ERS position paper. Eur Respir J 2004; 23: 932-946.

14 Balcells E, Antó JM, Gea J, et al. Characteristics of patients admitted for the first time for COPD exacerbation. Respir Med 2009; 103: 1293-1302.

15 Garcia-Aymerich J, Gómez FP, Benet M, et al. Identification and prospective validation of clinically relevant chronic obstructive pulmonary disease (COPD) subtypes. Thorax 2011; 66: 430-437.

16 Donaire-Gonzalez D, Gimeno-Santos E, Serra I, et al. Validación del cuestionario de actividad física de Yale en pacientes con enfermedad pulmonar obstructiva crónica. [Validation of the Yale physical activity survey in chronic obstructive pulmonary disease patients.] Arch Bronconeumol 2011; 47: 552-560.

17 Patel SA, Benzo RP, Slivka WA, et al. Activity monitoring and energy expenditure in COPD patients: a validation study. COPD 2007; 4: 107-112.

18 Watz H, Waschki B, Meyer T, et al. Physical activity in patients with COPD. Eur Respir J 2009; 33: 262-272.

19 Howley ET. Type of activity: resistance, aerobic and leisure versus occupational physical activity. Med Sci Sports Exerc 2001; 33: Suppl. 6, S364-S369.

20 Strath SJ, Pfeiffer KA, Whitt-Glover MC. Accelerometer use with children, older adults, and adults with functional limitations. Med Sci Sports Exerc 2012; 44: S77-S85.

21 Dipietro L, Caspersen CJ, Ostfeld AM, et al. A survey for assessing physical activity among older adults. Med Sci Sports Exerc 1993; 25: 628-642.

22 Troosters T, Sciurba F, Battaglia S, et al. Physical inactivity in patients with COPD, a controlled multi-center pilotstudy. Respir Med 2010; 104: 1005-1011.

23 Pitta F, Troosters T, Spruit MA, et al. Characteristics of physical activities in daily life in chronic obstructive pulmonary disease. Am J Respir Crit Care Med 2005; 171: 972-927.

24 De Abajo S, Larriba R, Marquez S. Validity and reliability of the Yale Physical Activity Survey in Spanish elderly. J Sports Med Phys Fitness 2001; 41: 479-485.

25 King AC, Satariano WA, Marti J, et al. Multilevel modeling of walking behavior: advances in understanding the interactions of people, place, and time. Med Sci Sports Exerc 2008; 40: Suppl. 7, S584-S593.

26 Sisson SB, Katzmarzyk PT. International prevalence of physical activity in youth and adults. Obes Rev 2008; 9: 606-614.

27 Hospes G, Bossenbroek L, Ten Hacken NHT, et al. Enhancement of daily physical activity increases physical fitness of outclinic COPD patients: results of an exercise counseling program. Patient Educ Couns 2009; 75: 274-278.

28 Jakicic JM, Wing RR, Butler BA, et al. Prescribing exercise in multiple short bouts versus one continuous bout: effects on adherence, cardiorespiratory fitness, and weight loss in overweight women. Int J Obes Relat Metab Disord 1995; 19: 893-901.

29 Murtagh EM, Boreham CAG, Nevill A, et al. The effects of 60 minutes of brisk walking per week, accumulated in two different patterns, on cardiovascular risk. Prev Med 2005; 41: 92-97.

30 Schmidt WD, Biwer CJ, Kalscheuer LK. Effects of long versus short bout exercise on fitness and weight loss in overweight females. J Am Coll Nutr 2001; 20: 494-501.

31 Pitta F, Troosters T, Probst VS, et al. Are patients with COPD more active after pulmonary rehabilitation? Chest 2008; 134: 273-280.

32 Haskell WL, Lee IM, Pate RR, et al. Physical activity and public health: updated recommendation for adults from the American College of Sports Medicine and the American Heart Association. Med Sci Sports Exerc 2007; 39: 1423-1434.

33 US Dept of Health and Human Services. 2008 physical activity guidelines for Americans: be active, healthy, and happy! Washington, US Dept of Health and Human Services, 2008. Available from www.health.gov/paguidelines/ pdf/paguide.pdf 